\title{
BMJ Open Trends in vital signs and routine biomarkers in patients with sepsis during resuscitation in the emergency department: a prospective observational pilot study
}

\author{
Vincent M Quinten, ${ }^{1}$ Matijs van Meurs, ${ }^{2,3}$ Jan C ter Maaten, ${ }^{1}$ Jack J M Ligtenberg ${ }^{1}$
}

To cite: Quinten VM, van Meurs M, ter Maaten JC, et al. Trends in vital signs and routine biomarkers in patients with sepsis during resuscitation in the emergency department: a prospective observational pilot study. BMJ Open 2016;6:e009718.

doi:10.1136/bmjopen-2015009718

- Prepublication history for this paper is available online. To view these files please visit the journal online (http://dx.doi.org/10.1136/ bmjopen-2015-009718).

Received 14 August 2015 Revised 8 March 2016 Accepted 21 April 2016

${ }^{1}$ Department of Emergency Medicine, University Medical Center Groningen, Groningen, The Netherlands

${ }^{2}$ Department of Critical Care, University Medical Center Groningen, Groningen, The Netherlands

${ }^{3}$ Department of Pathology and Medical Biology, Medical Biology Section, University Medical Center Groningen, Groningen, The Netherlands

Correspondence to Vincent M Quinten; v.m.quinten@umcg.nl

\section{ABSTRACT}

Objectives: Sepsis lacks a reliable and readily available measure of disease activity. Thereby, it remains unclear how to monitor response to treatment. Research on numerous (new) biomarkers associated with sepsis provided disappointing results and little is known about changes in vital signs during sepsis resuscitation. We hypothesised that trends in vital signs together with routine biomarker levels during resuscitation might provide information about the response to treatment at a very early stage of sepsis in the emergency department (ED). We therefore explore trends in vital signs and routine biomarker levels during sepsis resuscitation in the ED.

Design: Prospective observational pilot study. Setting: ED of a tertiary care teaching hospital. Participants: 99 Adult non-trauma patients with suspected infection and 2 or more systemic inflammatory response syndrome criteria admitted to the ED.

\section{Primary and secondary outcome measures:} Vital signs and biomarker levels at admittance (TO) and after $3 \mathrm{~h}$ in the ED (T1).

Results: In total, data of 99 patients were analysed. Of these patients, 63 presented with sepsis, 30 with severe sepsis and 6 with septic shock. All vital signs decreased, except for peripheral oxygen saturation which increased. Almost all routine biomarker levels decreased during resuscitation, except for $C$ reactive protein, bands, potassium, troponin $\mathrm{T}$ and direct bilirubin which remained stable. Sodium, chloride and $\mathrm{N}$-terminal prohormone of brain natriuretic peptide increased slightly.

Conclusions: Vital signs and biomarker levels showed descending trends during resuscitation, except for parameters directly affected by treatment modalities. Despite these trends, most patients improved clinically. Trends in vital signs and routine biomarkers might be helpful in predicting clinical course and response to treatment in patients with sepsis during early resuscitation.

\section{Strengths and limitations of this study}

- Our pilot study is the first to look at trends in vital signs and biomarker levels during sepsis resuscitation in the emergency department.

- Our pilot study shows that there are significant trends in vital signs and biomarker levels during resuscitation, and that these trends might potentially serve as a guide for treatment or to measure disease activity.

- Our pilot study was not designed to find the cause of the trends: trends might or might not have evolved as a result of the treatment provided.

- The measurement interval of $3 \mathrm{~h}$ chosen in this study might not be the optimal one; we recommend a follow-up study to find the optimal interval between measurements.

\section{INTRODUCTION}

Early and aggressive resuscitation is an important factor to reduce mortality of sepsis. ${ }^{12}$ It appears that early recognition of patients with sepsis and timely and aggressive resuscitation are more important than the specific kind of treatment provided. ${ }^{1} \quad 3 \quad 4$ Sepsis lacks a reliable measure of disease activity, similar to the viral load in HIV or left ventricle function in cardiology. ${ }^{5} \quad 6$ Therefore, it remains unclear how response to treatment can be monitored. ${ }^{6}{ }^{7}$ One known approach to monitor this is to monitor the patient's vital signs. However, there is little information about changes in vital signs in sepsis and their relation to treatment during early resuscitation in the emergency department (ED). Furthermore, numerous biomarkers associated with sepsis have been studied for this purpose, generally with disappointing results. Their sensitivity 
and specificity are too low to be of real clinical value and they are often not readily available. ${ }^{78}$

Up to $50 \%$ of all patients with sepsis are admitted through the ED. ${ }^{9}$ Patients are usually transferred from the ED to either the intensive care unit (ICU) or nursing wards within $4 \mathrm{~h}^{10}{ }^{11}$ Within these $4 \mathrm{~h}$, early resuscitation is initiated, preferably as soon as possible. ${ }^{6}$ We hypothesise that trends in vital signs together with routine biomarker levels during the resuscitation of patients with sepsis in the ED might provide information about the response to treatment. This information is useful to guide treatment at a very early stage of sepsis, while the patient is still in the ED. The response to treatment could be used to tailor the patient's treatment and monitoring and, at the same time, prevent doing harm to patients with mild sepsis with too aggressive treatment. It could furthermore serve as a feasible and accurate way to recognise the patients with a great chance to deteriorate and potentially provide an early warning of deterioration. ${ }^{12}$ To the best of our knowledge, there are no data available about trends in vital signs and biomarkers during resuscitation in the ED. Therefore, we performed a pilot study within the $4 \mathrm{~h}$ time frame that the patient is in the ED.

\section{METHODS}

\section{Study design and setting}

We performed a prospective observational pilot study in the ED of the University Medical Center Groningen, a tertiary care teaching hospital with over 34000 visits to the ED annually. The pilot study was aimed to establish power calculations and feasibility of a full-scale study on the use of trends in vital signs and biomarkers as response to treatment parameter. The pilot aimed to include a convenience sample of 100 patients within a limited 6-month time frame. Data were collected between October 2013 and April 2014. To prevent selection bias, taking blood samples in patients with an altered mental status due to sepsis was also approved by the review board. In these cases, informed consent was obtained from the next of kin or from the patient during their stay in hospital.

\section{Study population and protocol}

Adult non-trauma patients visiting the ED with presumed infection or sepsis were screened for inclusion. Inclusion criteria were: age $\geq 18$ years, presumed or confirmed infection, and two or more systemic inflammatory response syndrome (SIRS) criteria as defined by the International Sepsis Definitions Conference. ${ }^{13}$

Patients are usually transferred from the ED to either the ICU or nursing ward within $4 \mathrm{~h}$. To detect trends in vital signs and biomarker levels, we took measurements at two points within this time frame: at admittance to the ED $\left(\mathrm{T}_{0}\right)$ and after $3 \mathrm{~h}\left(\mathrm{~T}_{1}\right)$. At $\mathrm{T}_{0}$, a nurse measured the patient's vital signs and took a routine blood sample. Vital signs were measured with a patient monitor
(IntelliVue MP30 System with Multi-Measurement Module, Philips, Eindhoven, The Netherlands), except for temperature which was measured using an electronic tympanic ear thermometer (Genius 2; Mountainside Medical Equipment, Marcy, New York, USA). Simultaneously with the routine blood sample, the nurse took a number of additional blood vials for this study. These additional vials were temporarily stored until informed consent was obtained. This procedure ensured that treatment was not delayed for patients participating in the study. The vials for the routine blood sample were immediately sent to the hospital's central laboratory and were analysed for the routine biomarkers listed in table 1.

Patients or their healthcare proxies had to provide written informed consent before $\mathrm{T}_{1}$; otherwise, the patient was excluded from the study and the stored vials were destroyed. The stored vials were sent to the central laboratory for analysis immediately after obtaining informed consent. The blood in these vials was analysed for four additional routinely available biomarkers, as shown in table 1 . These biomarkers were added for the following reasons: N-terminal prohormone of brain natriuretic peptide (NT pro-BNP) as a marker for fluid overload, cortisol as a marker for stress response, D-dimer for coagulation status and marker of disseminated intravascular coagulation and troponin $\mathrm{T}$ as a marker of myocardial damage.

At $\mathrm{T}_{1}$, new blood samples were collected and immediately analysed for all biomarkers shown in table 1 . The patient's vital signs were also recorded at $T_{1}$ using the same procedures and equipment as at $\mathrm{T}_{0}$. Furthermore, we recorded the amount of intravenous fluids given to the patient in the ED until $\mathrm{T}_{1}$. In case a patient was transferred to the ICU or a ward before $\mathrm{T}_{1}$, a researcher took the $\mathrm{T}_{1}$ blood samples and vital signs there according to the study protocol.

The attending physician was asked for the suspected focus of sepsis at the moment the patient was transferred out of the ED and was allowed to select multiple options. Demographic data were collected from the patient's electronic medical records. All patients received treatment according to the routine sepsis protocol, including fluid resuscitation, antibiotics and supplemental oxygen. According to protocol, fluid resuscitation was performed by an initial fluid challenge of $500 \mathrm{~mL}$ saline solution $(\mathrm{NaCl} 0.9 \%)$ in $10 \mathrm{~min}$, followed by $500 \mathrm{~mL}$ every $15 \mathrm{~min}$ until a mean arterial pressure (MAP) of $\geq 65 \mathrm{~mm} \mathrm{Hg}$ was reached. ${ }^{6}$ When the MAP was still $<65 \mathrm{~mm} \mathrm{Hg}$ after $2 \mathrm{~L}$ of saline, an intensivist was consulted to transfer the patient to the ICU and start inotropic medication. From previous studies in our department, we know that the median time to start fluid resuscitation was $21 \mathrm{~min}$ (sepsis 26, severe sepsis 15 and septic shock $4 \mathrm{~min}$ ). Antibiotics were given in accordance with the guidelines provided by the Dutch Working Party on Antibiotic Policy (SWAB). ${ }^{14}$ The median time to intravenous antibiotics was $61 \mathrm{~min}$ 
Table 1 Overview of the measured biomarkers and their characteristics

\begin{tabular}{|c|c|c|c|}
\hline Name & Unit & CV (\%) & Reference values \\
\hline \multicolumn{4}{|l|}{ Routine biomarkers } \\
\hline Albumin & $\mathrm{g} / \mathrm{L}$ & 1.4 & $35-50$ \\
\hline ALP & $\mathrm{U} / \mathrm{L}$ & 2.2 & Male: $<115$, female: $<98$ \\
\hline AST & $\mathrm{U} / \mathrm{L}$ & 1.4 & Male: $<35$, female: $<31$ \\
\hline Bands & $\%$ & $\mathrm{n} / \mathrm{a}$ & $0-3$ \\
\hline Bilirubin, direct & $\mu \mathrm{mol} / \mathrm{L}$ & 1.9 & $<5$ \\
\hline Bilirubin, total & $\mu \mathrm{mol} / \mathrm{L}$ & 1.9 & $<17$ \\
\hline Calcium & $\mathrm{mmol} / \mathrm{L}$ & 1.4 & $2.20-2.60$ \\
\hline Chloride & $\mathrm{mmol} / \mathrm{L}$ & 0.8 & $97-107$ \\
\hline Creatinine & $\mu \mathrm{mol} / \mathrm{L}$ & 2.0 & Male: $50-110$, female: $50-90$ \\
\hline CRP & $\mathrm{mg} / \mathrm{L}$ & 3.0 & $<5$ \\
\hline$\gamma$-GT & $\mathrm{U} / \mathrm{L}$ & 1.9 & Male: $<55$, female: $<38$ \\
\hline Glucose & $\mathrm{mmol} / \mathrm{L}$ & 1.5 & $4.0-5.5$ (fastening) \\
\hline $\mathrm{Hb}$ & $\mathrm{mmol} / \mathrm{L}$ & 1.3 & Male: $8.7-10.6$, female: $7.5--9.9$ \\
\hline Lactate & $\mathrm{mmol} / \mathrm{L}$ & 1.5 & $0.5-2.2$ \\
\hline $\mathrm{LDH}$ & $\mathrm{U} / \mathrm{L}$ & 1.3 & Male: $<248$, female: $<247$ \\
\hline Leucocytes & $10^{9} / \mathrm{L}$ & 1.8 & $4-10$ \\
\hline Potassium & $\mathrm{mmol} / \mathrm{L}$ & 0.8 & $3.5-5.0$ \\
\hline Sodium & $\mathrm{mmol} / \mathrm{L}$ & 0.7 & $135-145$ \\
\hline Thrombocytes & $10^{9} / \mathrm{L}$ & 4.4 & $150-350$ \\
\hline Urea & $\mathrm{mmol} / \mathrm{L}$ & 2.5 & $2.5-7.5$ \\
\hline \multicolumn{4}{|c|}{ Study-specific additional biomarkers } \\
\hline \multirow[t]{3}{*}{ Cortisol } & $\mathrm{nmol} / \mathrm{L}$ & 3.8 & 08:00:200-800 \\
\hline & & & $16: 00: 100-400$ \\
\hline & & & 22:00:50-200 \\
\hline D-dimer & $\mathrm{ng} / \mathrm{mL}$ & 4.5 & $<500$ \\
\hline hs-Trop T & $\mathrm{ng} / \mathrm{L}$ & 5.0 & $<14$ \\
\hline NT pro-BNP & $\mathrm{ng} / \mathrm{L}$ & 2.2 & $<175,>75$-year: <450 \\
\hline
\end{tabular}

(sepsis 75, severe sepsis 54 and septic shock $45 \mathrm{~min}$ ) from ED entrance in previous sepsis studies in our department. Supplemental oxygen was given to maintain an $\mathrm{SaO} 2$ between $94 \%$ and $98 \%$. The treatment protocol did not change during the inclusion period of the study.

\section{Statistical methods}

Continuous data are presented as mean with SD or median with IQR depending on their distribution. Normality was tested using the Shapiro-Wilk test for normality. Categorical data are presented as absolute numbers with percentages. The Wilcoxon-related samples signed rank test was used for comparison of biomarker levels and vital signs between $T_{0}$ and $T_{1}$. Effect sizes are presented as Cohen's $d .{ }^{15}$ The variance between the sepsis severity groups and effect of medication or comorbidities on the response to treatment was tested using the non-parametric Jonckheere-Terpstra test. Missing data were excluded for analysis. All statistical analyses were performed using IBM SPSS Statistics for Windows V.22.0 (IBM Corp, Armonk, New York, USA). A p value of $\leq 0.05$ was considered significant; all tests were two tailed.

\section{RESULTS}

In total, 101 patients were included. Two patients were excluded since informed consent could not be obtained from one patient before $\mathrm{T}_{1}$ and was withdrawn by another patient. The remaining 99 patients were included in the final analysis. Of these patients, 63 presented with sepsis, 30 with severe sepsis and 6 with septic shock at ED admission. Patient characteristics, including comorbidities and medication use prior to ED presentation, are shown in table 2. Patients with severe sepsis more frequently had a history of mild liver disease $(p=0.02)$. Patients with sepsis used diuretics more often $(p=0.02)$. The presumed focus of infection, vital signs and treatment parameters are shown in table 3. The most frequent foci were pulmonic and urogenital. The frequency of these foci did not differ between severity groups. Patients in the septic shock group received more intravenous fluids (3.5 L; IQR 2.9-5.0 L) compared with those with severe sepsis and those with sepsis $(\mathrm{p}=0.009)$.

\section{Vital signs}

Blood pressure at $T_{0}$ and $T_{1}$ was inversely related to sepsis severity as blood pressure decreased with 
Table 2 Patient characteristics, comorbidity, medication at presentation in the emergency department

\begin{tabular}{|c|c|c|c|c|c|c|}
\hline & $\begin{array}{l}N \\
99\end{array}$ & $\begin{array}{l}\text { Overall } \\
99(100.0 \%)\end{array}$ & $\begin{array}{l}\text { Sepsis } \\
63(63.6 \%)\end{array}$ & $\begin{array}{l}\text { Severe sepsis } \\
30(30.3 \%)\end{array}$ & $\begin{array}{l}\text { Septic shock } \\
6(6.1 \%)\end{array}$ & p Value \\
\hline \multicolumn{7}{|l|}{ Demographics } \\
\hline Age† & 99 & $59(47-70)$ & $60(49-70)$ & $56(44.5-73.3)$ & $56.5(47-68.8)$ & 0.50 \\
\hline \multicolumn{7}{|l|}{ Gender } \\
\hline Male* & 99 & $57(57.6 \%)$ & $29(46.0 \%)$ & $23(76.7 \%)$ & $5(83.3 \%)$ & 1.00 \\
\hline Female* & 99 & $42(42.4 \%)$ & $34(54.0 \%)$ & $7(23.3 \%)$ & $1(16.7 \%)$ & 1.00 \\
\hline \multicolumn{7}{|l|}{ Comorbidity } \\
\hline Myocardial infarction* & 99 & $13(13.1 \%)$ & $11(17.5 \%)$ & $2(6.7 \%)$ & $0(0 \%)$ & 0.08 \\
\hline Congestive heart failure* & 99 & $6(6.1 \%)$ & $5(7.9 \%)$ & $1(3.3 \%)$ & $0(0 \%)$ & 0.29 \\
\hline Peripheral vascular disease* & 99 & $6(6.1 \%)$ & $4(6.3 \%)$ & $2(6.7 \%)$ & $0(0 \%)$ & 0.80 \\
\hline Cerebrovascular disease ${ }^{*}$ & 98 & $12(12.1 \%)$ & $9(14.3 \%)$ & $2(6.7 \%)$ & $1(16.7 \%)$ & 0.44 \\
\hline Dementia* $^{*}$ & 99 & $3(3.0 \%)$ & $2(3.2 \%)$ & $1(3.3 \%)$ & $0(0 \%)$ & 0.86 \\
\hline Chronic pulmonary disease* & 99 & $23(23.2 \%)$ & $17(27.0 \%)$ & $14(13.3 \%)$ & $2(33.3 \%)$ & 0.33 \\
\hline Connective tissue disease ${ }^{\star}$ & 99 & $0(0 \%)$ & $0(0 \%)$ & $0(0 \%)$ & $0(0 \%)$ & 1.00 \\
\hline Ulcer disease ${ }^{\star}$ & 99 & $2(2.0 \%)$ & $1(1.6 \%)$ & $1(3.3 \%)$ & $0(0 \%)$ & 0.76 \\
\hline Mild liver disease* & 99 & $11(11.1 \%)$ & $3(4.8 \%)$ & $8(26.7 \%)$ & $0(0 \%)$ & $0.02 \ddagger$ \\
\hline Diabetes* & 99 & $16(16.2 \%)$ & $12(19.0 \%)$ & $4(13.3 \%)$ & $0(0 \%)$ & 0.25 \\
\hline Hemiplegia* & 99 & $2(2.0 \%)$ & $1(1.6 \%)$ & $1(3.3 \%)$ & $0(0 \%)$ & 0.76 \\
\hline Moderate or severe renal disease ${ }^{*}$ & 99 & $23(23.2 \%)$ & $11(17.5 \%)$ & $10(33.3 \%)$ & 2 (33.3\%) & 0.08 \\
\hline Diabetes with end-organ damage* & 99 & $3(3.0 \%)$ & $2(3.2 \%)$ & $1(3.3 \%)$ & $0(0 \%)$ & 0.86 \\
\hline Any tumour* & 99 & $30(30.3 \%)$ & $20(31.7 \%)$ & $9(30.0 \%)$ & $1(16.7 \%)$ & 0.60 \\
\hline Leukaemia* & 99 & $5(5.1 \%)$ & $3(4.8 \%)$ & $2(6.7 \%)$ & $1(16.7 \%)$ & 0.92 \\
\hline Lymphoma* & 99 & $7(7.1 \%)$ & $6(9.5 \%)$ & $1(3.3 \%)$ & $0(0 \%)$ & 0.20 \\
\hline Moderate or severe liver disease ${ }^{*}$ & 99 & $4(4.0 \%)$ & $1(1.6 \%)$ & $3(10.0 \%)$ & $0(0 \%)$ & 0.16 \\
\hline Metastatic solid tumour* & 99 & $8(8.1 \%)$ & $6(9.5 \%)$ & $2(6.7 \%)$ & $0(0 \%)$ & 0.44 \\
\hline AIDS $^{*}$ & 99 & $0(0 \%)$ & $0(0 \%)$ & $0(0 \%)$ & $0(0 \%)$ & 1.00 \\
\hline Charlson Index $†$ & 99 & $2(1-4)$ & $2(1-4)$ & $2(2-4)$ & $1(1-2)$ & 0.80 \\
\hline \multicolumn{7}{|c|}{ Medication at emergency department presentation } \\
\hline RAS inhibitor* & 99 & $25(25.3 \%)$ & $16(25.4 \%)$ & $5(16.7 \%)$ & $4(66.7 \%)$ & 0.70 \\
\hline$\beta$-blocker* & 99 & $34(34.3 \%)$ & $21(33.3 \%)$ & $10(33.3 \%)$ & $3(50.0 \%)$ & 0.69 \\
\hline Calcium-channel blocker* & 99 & $15(15.2 \%)$ & $12(19.0 \%)$ & $2(6.7 \%)$ & $1(16.7 \%)$ & 0.19 \\
\hline Antibiotic ${ }^{*}$ & 99 & $30(30.3 \%)$ & $19(30.2 \%)$ & $10(33.3 \%)$ & $1(16.7 \%)$ & 0.93 \\
\hline Immunosuppressive medication* & 99 & $33(33.3 \%)$ & $19(30.2 \%)$ & $13(43.3 \%)$ & $1(16.7 \%)$ & 0.51 \\
\hline Diuretic $^{*}$ & 99 & $24(24.2 \%)$ & $20(31.7 \%)$ & $4(13.3 \%)$ & $0(0 \%)$ & $0.02 \ddagger$ \\
\hline Non-steroid anti-inflammatory drug* & 99 & $32(32.3 \%)$ & $23(36.5 \%)$ & $9(30.0 \%)$ & $0(0 \%)$ & 0.16 \\
\hline Paracetamol $^{\star}$ & 99 & $18(18.2 \%)$ & $11(17.5 \%)$ & $7(23.3 \%)$ & $0(0 \%)$ & 0.99 \\
\hline Antidiabetic medication* & 99 & $17(17.2 \%)$ & $13(20.6 \%)$ & $4(13.3 \%)$ & $0(0 \%)$ & 0.19 \\
\hline \multicolumn{7}{|l|}{$\begin{array}{l}\text { Data are presented as: } \\
\text { *Absolute number and percentage. } \\
\text { †Median and IQR. } \\
\text { †Significant result. } \\
\text { RAS, renin angiotensin system. }\end{array}$} \\
\hline
\end{tabular}

increasing severity of sepsis. The results of all vital sign measurements are shown in tables 3 and 4 . Table 2 shows the vital signs for $T_{0}$ and $T_{1}$ separated by the sepsis severity groups. Table 4 includes the $\delta$ 's between $\mathrm{T}_{0}$ and $\mathrm{T}_{1}$ for each vital sign; these are also graphically represented in figure 1 . We found significant differences for all measured vital signs. As becomes apparent from figure 1, all vital signs decreased during the measurement time frame, except for peripheral oxygen saturation which increased by $1.1 \%$. The heart rate and respiratory rate dropped by $>10 \%$ during resuscitation $(\mathrm{p}<0.001)$. At the same time, the systolic blood pressure decreased by $5 \%$ and diastolic blood pressure decreased by $>9 \%(\mathrm{p}<0.001)$.

\section{Biomarkers}

The results for the biomarker levels are shown in table 4 , including $\delta$ 's between $\mathrm{T}_{0}$ and $\mathrm{T}_{1}$ for each biomarker. These $\delta$ 's are also shown in figure 2. Almost all routine biomarkers levels decreased during resuscitation in the $\mathrm{ED}$, except for $\mathrm{C}$ reactive protein (CRP), bands, potassium and direct bilirubin which remained stable. Levels of sodium and chloride increased slightly by $0.8 \%$ and $2.1 \% \quad(p<0.001)$, respectively. The levels of NT pro-BNP increased by $3.0 \% \quad(p=0.039)$ during resuscitation. Cortisol and D-dimer levels decreased by $20.1 \%$ $(p<0.001)$ and $3.7 \% \quad(p=0.039)$, respectively. The highsensitivity troponin $\mathrm{T}$ (hs-Trop $\mathrm{T}$ ) levels did not show a significant trend. 
Table 3 Presumed focus, vital signs and treatment parameters in the emergency department

\begin{tabular}{|c|c|c|c|c|c|c|}
\hline & $\begin{array}{l}N \\
99\end{array}$ & $\begin{array}{l}\text { Overall } \\
99(100.0 \%)\end{array}$ & $\begin{array}{l}\text { Sepsis } \\
63(63.6 \%)\end{array}$ & $\begin{array}{l}\text { Severe sepsis } \\
30(30.3 \%)\end{array}$ & $\begin{array}{l}\text { Septic shock } \\
6(6.1 \%)\end{array}$ & p Value \\
\hline \multicolumn{7}{|l|}{ Presumed focus } \\
\hline Respiratory* & 99 & $49(49.5 \%)$ & $31(49.2 \%)$ & $13(43.3 \%)$ & $5(83.3 \%)$ & 0.71 \\
\hline Urogenital $^{*}$ & 99 & 31 (31.3\%) & $21(33.3 \%)$ & $8(26.7 \%)$ & $2(33.3 \%)$ & 0.61 \\
\hline Skin/soft-tissue/wound* & 99 & $6(6.1 \%)$ & $4(6.3 \%)$ & $2(6.7 \%)$ & $0(0.0 \%)$ & 0.80 \\
\hline Intra-abdominal $^{\star}$ & 99 & $21(21.2 \%)$ & $13(20.6 \%)$ & $6(20.0 \%)$ & $2(33.3 \%)$ & 0.76 \\
\hline Catheter/tube/implant* & 99 & $3(3.0 \%)$ & $2(3.2 \%)$ & $1(3.3 \%)$ & $0(0.0 \%)$ & 0.86 \\
\hline Meningitis* & 99 & $1(1.0 \%)$ & $1(1.6 \%)$ & $0(0.0 \%)$ & $0(0.0 \%)$ & 0.46 \\
\hline Other or unknown focus* & 99 & $15(15.2 \%)$ & $10(15.9 \%)$ & $4(13.3 \%)$ & $1(16.7 \%)$ & 0.82 \\
\hline \multicolumn{7}{|l|}{ Vital signs } \\
\hline $\mathrm{T}_{0}$ : Heart rate $(\mathrm{bpm}) \dagger$ & 99 & $110(100-120)$ & $110(100-120)$ & $112.5(104.5-120.8)$ & $113.5(93.5-136.8)$ & 0.66 \\
\hline $\mathrm{T}_{1}:$ Heart rate $(\mathrm{bpm}) \dagger$ & 93 & $98(90-108.5)$ & $98(89-110)$ & $100(94.3-105)$ & $100(88.8-138)$ & 0.71 \\
\hline $\mathrm{T}_{0}$ : Systolic blood pressure $(\mathrm{mm} \mathrm{Hg}) \ddagger$ & 99 & $124.1 \pm 21.87$ & $128.9 \pm 18.96$ & $123.3 \pm 17.63$ & $78.2 \pm 17.00$ & $0.002 \S$ \\
\hline $\mathrm{T}_{1}$ : Systolic blood pressure $(\mathrm{mm} \mathrm{Hg}) \ddagger$ & 91 & $115.4 \pm 19.09$ & $119.7 \pm 17.84$ & $112.3 \pm 17.41$ & $89.8 \pm 17.66$ & $0.002 \S$ \\
\hline $\mathrm{T}_{0}$ : Diastolic blood pressure $(\mathrm{mm} \mathrm{Hg}) \ddagger$ & 99 & $71.5 \pm 15.58$ & $73.4 \pm 14.48$ & $71.7 \pm 15.63$ & $50.2 \pm 12.22$ & $0.03 \S$ \\
\hline $\mathrm{T}_{1}$ : Diastolic blood pressure $(\mathrm{mm} \mathrm{Hg}) \ddagger$ & 91 & $64.6 \pm 13.32$ & $65.3 \pm 12.50$ & $64.9 \pm 15.46$ & $55.5 \pm 7.01$ & 0.27 \\
\hline $\mathrm{T}_{0}: \mathrm{MAP}(\mathrm{mm} \mathrm{Hg}) \ddagger$ & 99 & $89.2 \pm 15.98$ & $91.9 \pm 14.01$ & $89.4 \pm 14.71$ & $59.67 \pm 13.32$ & $0.02 \S$ \\
\hline $\mathrm{T}_{1}: \mathrm{MAP}(\mathrm{mm} \mathrm{Hg}) \ddagger$ & 91 & $81.5 \pm 14.12$ & $83.5 \pm 13.05$ & $80.3 \pm 15.38$ & $67.2 \pm 10.23$ & $0.02 \S$ \\
\hline $\mathrm{T}_{0}$ : Respiration rate (breath/min) $\dagger$ & 93 & $23(18-28)$ & $23(18-27.3)$ & $23(18-27.5)$ & $29(21-34.8)$ & 0.31 \\
\hline $\mathrm{T}_{1}$ : Respiration rate (breath/min) $\dagger$ & 86 & $20(17.8-24)$ & $20(18-27)$ & $20(18.8-24)$ & $24(14.3-34.3)$ & 0.27 \\
\hline $\mathrm{T}_{0}:$ Oxygen saturation $(\%) \dagger$ & 98 & $96(93-98)$ & $95(93-98)$ & $96(92.8-98)$ & $94(86.5-98)$ & 0.88 \\
\hline $\mathrm{T}_{1}$ : Oxygen saturation (\%)† & 89 & $97(95-98.5)$ & $97(96-98)$ & 97 (95.3-99) & $96(93.5-97.3)$ & 0.72 \\
\hline $\mathrm{T}_{0}:$ Temperature $\left({ }^{\circ} \mathrm{C}\right) \dagger$ & 99 & $38.4(37.5-38.9)$ & $38.4(37.7-38.9)$ & $38.6(37.8-39.0)$ & $36.9(34.5-38.8)$ & 0.58 \\
\hline $\mathrm{T}_{1}$ : Temperature $\left({ }^{\circ} \mathrm{C}\right) \dagger$ & 91 & $37.7(36.8-38.6)$ & $37.7(37.1-38.5)$ & $37.7(36.6-38.8)$ & $36.6(36.6-39.2)$ & 0.60 \\
\hline \multicolumn{7}{|l|}{ Treatment parameters } \\
\hline Intravenous fluids $(\mathrm{L}) \dagger$ & 98 & $1.0(0.5-2.0)$ & $1.0(0.5-1.5)$ & $1.0(0.9-2.0)$ & $3.5(2.9-5.0)$ & $0.009 \S$ \\
\hline $\mathrm{T}_{0}$ : Supplemental oxygen (L)† & 99 & $0.0(0.0-2.0)$ & $0.0(0.0-2.0)$ & $0.0(0.0-2.0)$ & $2.0(0.0-15.0)$ & 0.74 \\
\hline $\mathrm{T}_{1}$ : Supplemental oxygen $(\mathrm{L}) \dagger$ & 87 & $2.0(0.0-3.0)$ & $2.0(0.0-3.0)$ & $0.0(0.0-2.5)$ & $13.5(1.5-15.0)$ & 0.25 \\
\hline \multicolumn{7}{|l|}{$\begin{array}{l}\text { Data are presented as: } \\
\text { *Absolute number and percentage. } \\
\text { †Median and IQR. } \\
\text { †Mean } \pm \text { SD. } \\
\text { §Significant result. } \\
\text { MAP, mean arterial pressure. }\end{array}$} \\
\hline
\end{tabular}


Table 4 The $\delta$ in vital signs and biomarker levels between $T_{0}$ and $T_{1}$

\begin{tabular}{|c|c|c|c|c|c|c|c|}
\hline & $\mathbf{N}$ & TO & $\mathbf{N}$ & T1 & $\delta(\mathrm{T} 1-\mathrm{T} 0)$ & p Value & d \\
\hline \multicolumn{8}{|l|}{ Vital signs } \\
\hline Heart rate $(\mathrm{bpm}) \dagger$ & 99 & 110 (100 to 120$)$ & 93 & 98 (90 to 108.5$)$ & $-10(-17.5$ to -4.0$)$ & $<0.001 \S$ & -0.75 \\
\hline Systolic blood pressure $(\mathrm{mm} \mathrm{Hg})^{*}$ & 99 & $124.1 \pm 21.87$ & 91 & $115.4 \pm 19.09$ & $-7.5 \pm 19.02$ & $<0.001 \S$ & -0.38 \\
\hline Diastolic blood pressure $(\mathrm{mm} \mathrm{Hg})^{*}$ & 99 & $71.5 \pm 15.58$ & 91 & $64.6 \pm 13.32$ & $-6.4 \pm 13.37$ & $<0.001 \S$ & -0.44 \\
\hline $\mathrm{MAP}(\mathrm{mm} \mathrm{Hg})^{*}$ & 99 & $89.2 \pm 15.98$ & 91 & $81.5 \pm 14.12$ & $7.0 \pm 13.86$ & $<0.001 \S$ & -0.46 \\
\hline Respiration rate (breath/min) $†$ & 93 & 23 (18 to 28$)$ & 86 & 20 (17.8 to 24$)$ & $-2(-6$ to -2$)$ & $0.003 \S$ & -0.32 \\
\hline Oxygen saturation $(\%) \dagger$ & 98 & 96 (93 to 98$)$ & 89 & 97 (95 to 98.5$)$ & $1.0(-1.0$ to 5.0$)$ & $0.001 \S$ & -0.35 \\
\hline Supplemental oxygen (L)† & 99 & $0.0(0.0$ to 2.0$)$ & 87 & $0.0(0.0$ to 3.0$)$ & 0.0 (0.0 to 2.0$)$ & $<0.001 \S$ & -0.40 \\
\hline Temperature $\left({ }^{\circ} \mathrm{C}\right) \dagger$ & 99 & 38.4 (37.5 to 38.9$)$ & 91 & 37.7 (36.8 to 38.6$)$ & $-0.4(-1.0$ to 0.3$)$ & $<0.001 \S$ & -0.41 \\
\hline \multicolumn{8}{|l|}{ Routine biomarkers } \\
\hline Albumin (g/L)† & 93 & 37 (35 to 40.5$)$ & 98 & 34 (31.8 to 37$)$ & $-3.0(-5.0$ to -1.0$)$ & $<0.001 \S$ & -0.78 \\
\hline ALP $(U / L) \dagger$ & 97 & 83 (55 to 136$)$ & 98 & 71.5 (48.8 to 115.5$)$ & $-9.0(-14.8$ to -3.0$)$ & $<0.001 \S$ & -0.77 \\
\hline AST (U/L)† & 98 & 26 (20 to 38.3$)$ & 98 & 24 (18 to 36.3$)$ & $-2.0(-4.5$ to -0.0$)$ & $<0.001 \S$ & -0.56 \\
\hline Bands $(\%) \dagger$ & 82 & 0.0 (0.0 to 2.3$)$ & 97 & $0.0(0.0$ to 3.5$)$ & $0.0(0.0$ to 0.0$)$ & 0.72 & -0.04 \\
\hline Bilirubin, direct $(\mu \mathrm{mol} / \mathrm{L}) \dagger$ & 97 & $4(3$ to 8$)$ & 98 & $4(3$ to 9$)$ & $0.0(-1.0$ to 1.0$)$ & 0.36 & -0.09 \\
\hline Bilirubin, total $(\mu \mathrm{mol} / \mathrm{L}) \dagger$ & 97 & 12 (8 to 18$)$ & 98 & $11(8$ to 16$)$ & $-1.0(-2.0$ to 0.0$)$ & $<0.001 \S$ & -0.41 \\
\hline Calcium $(\mathrm{mmol} / \mathrm{L}) \dagger$ & 92 & 2.23 (2.14 to 2.30$)$ & 98 & 2.09 (1.98 to 2.20$)$ & $-0.1(-0.2$ to -0.08$)$ & $<0.001 \S$ & -0.78 \\
\hline Chloride $(\mathrm{mmol} / \mathrm{L}) \dagger$ & 92 & 100 (96 to 102) & 98 & 102 (99 to 106$)$ & $2.0(1.0$ to 4.0$)$ & $<0.001 \S$ & -0.78 \\
\hline Creatinine $(\mu \mathrm{mol} / \mathrm{L}) \dagger$ & 99 & 85 (64 to 123$)$ & 98 & 83.5 (63.5 to 128.3$)$ & $-1.5(-8.0$ to 4.0$)$ & $0.02 \S$ & -0.24 \\
\hline CRP (mg/L)† & 99 & 93 (36 to 201$)$ & 98 & 99.5 (45.8 to 184.3$)$ & $0.0(-12.3$ to 8.5$)$ & 0.45 & -0.08 \\
\hline$\gamma$-GT $(\mathrm{U} / \mathrm{L}) \dagger$ & 96 & 46 (26.5 to 106.5$)$ & 98 & 39.5 (24.8 to 92.8$)$ & $-4.0(-12.0$ to -1.0$)$ & $<0.001 \S$ & -0.70 \\
\hline Glucose $(\mathrm{mmol} / \mathrm{L}) \dagger$ & 99 & $7.1(6.1$ to 8.6$)$ & 97 & 6.7 (5.9 to 7.7 ) & $-0.5(-1.2$ to 0.3$)$ & $0.001 \S$ & -0.34 \\
\hline Haemoglobin $(\mathrm{mmol} / \mathrm{L}) \dagger$ & 99 & 7.9 (6.9 to 8.7$)$ & 99 & 7.3 (6.5 to 8.2$)$ & $-0.6(-0.8$ to -0.2$)$ & $<0.001 \S$ & -0.79 \\
\hline Lactate $(\mathrm{mmol} / \mathrm{L}) \dagger$ & 86 & $1.6(1.08$ to 2.1$)$ & 96 & $1.2(0.9$ to 1.7$)$ & $-0.2(-0.8$ to 0.1$)$ & $<0.001 \S$ & -0.39 \\
\hline LDH (U/L)† & 98 & $212(163$ to 257.5$)$ & 98 & 177 (142.5 to 232.8$)$ & $-21.0(-41.0$ to -8.0$)$ & $<0.001 \S$ & -0.69 \\
\hline Leucocytes $\left(10^{9} / \mathrm{L}\right) \dagger$ & 99 & 12.1 (8.4 to 20.4$)$ & 99 & $11.9(7.9$ to 17.0$)$ & $-0.6(-1.6$ to 0.4$)$ & $0.005 \S$ & -0.28 \\
\hline Potassium (mmol/L)† & 98 & 3.9 (3.5 to 4.3 ) & 97 & 3.8 (3.5 to 4.3$)$ & $0.0(-0.3$ to 0.2$)$ & 0.28 & -0.11 \\
\hline Sodium $(\mathrm{mmol} / \mathrm{L}) \dagger$ & 98 & 137 (133 to 139$)$ & 98 & $138(134$ to 140.3$)$ & $1.0(0.0$ to 3.0$)$ & $<0.001 \S$ & -0.59 \\
\hline Thrombocytes $\left(10^{9} / \mathrm{L}\right) \dagger$ & 99 & 208 (163 to 284$)$ & 99 & 188 (143 to 259$)$ & $-15.0(-29.0$ to -3.0$)$ & $<0.001 \S$ & -0.63 \\
\hline Urea $(\mathrm{mmol} / \mathrm{L}) \dagger$ & 99 & 7.2 (4.8 to 12.2$)$ & 98 & $6.8(4.1$ to 12.1$)$ & $-0.4(-0.8$ to -0.1$)$ & $<0.001 \S$ & -0.64 \\
\hline \multicolumn{8}{|l|}{ Study-specific additional biomarkers } \\
\hline Cortisol (nmol/L)† & 91 & 860 (505 to 1245$)$ & 90 & 765 (366.3 to 1150$)$ & $-127.5(-352.5$ to -20.0$)$ & $<0.001 \S$ & -0.43 \\
\hline D-dimer (ng/mL)† & 92 & 735 (354 to 2274) & 90 & 779 (357 to 2403 ) & $-44(-171$ to 23$)$ & $0.003 \S$ & -0.32 \\
\hline hs-Trop T (ng/L)† & 92 & 20 (8.3 to 39$)$ & 92 & 23 (8.3 to 40.8$)$ & $-0.5(-3.0$ to 3.0$)$ & 0.68 & -0.04 \\
\hline NT pro-BNP (ng/L)† & 93 & 409 (143 to 2036) & 91 & 483 (155 to 2788$)$ & $10.0(-21.0$ to 169.0$)$ & $0.04 \S$ & -0.22 \\
\hline
\end{tabular}

Data are presented as:

${ }^{*}$ Absolute number and percentage.

†Median and IQR.

$\ddagger$ Mean \pm SD.

$\S$ Significant result.

$\gamma$-GT, $\gamma$-glutamyl transferase; ALP, alkaline phosphatase; AST, aspartate transaminase; CRP, C reactive protein; hs-Trop T, high-sensitivity troponin T; LDH, lactate dehydrogenase; MAP, mean arterial pressure; NT pro-BNP, N-terminal prohormone of brain natriuretic peptide. 


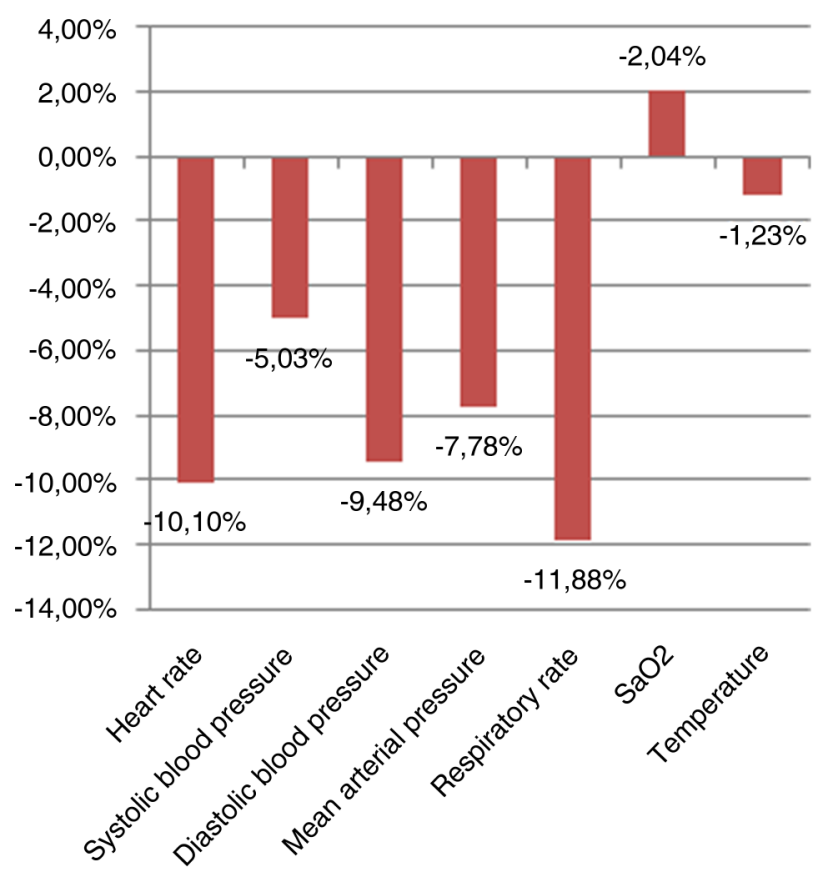

Figure 1 The $\delta$ in vital signs between $\mathrm{T}_{0}$ and $\mathrm{T}_{1}$. SaO2, peripheral oxygen saturation.

Biomarker levels were below the laboratory's lower detection limit in several instances. In these instances, their value was set to half the lower detection limit. Direct bilirubin levels were below the detection limit $(1.0 \mu \mathrm{mol} / \mathrm{L})$ in five cases at $\mathrm{T}_{0}$ and three cases at $\mathrm{T}_{1}$, D-dimer levels (detection limit: $150 \mathrm{ng} / \mathrm{mL}$ ) in five cases at $\mathrm{T}_{0}$ and seven cases at $\mathrm{T}_{1}$, hs-Trop $\mathrm{T}$ levels (detection limit: $3.0 \mathrm{ng} / \mathrm{L}$ ) in three cases at $\mathrm{T}_{0}$ and five cases at $\mathrm{T}_{1}$. During the calculation of the $\delta$ 's, the $\mathrm{T}_{0}$ values of the biomarkers were zero in a few cases. To avoid division by zero problems during the calculation of the percentual difference, these values were handled as missing data. Band levels were zero in five instances at $\mathrm{T}_{0}$ and in one instance the trombocyte level was zero at $\mathrm{T}_{0}$, these six instances have been excluded from analysis.

\section{Medication and comorbidity}

To explore confounding factors that might have affected the response to treatment, we analysed associations between medication use at ED presentation, comorbidity and the measured vital signs and biomarker levels. The use of antihypertensive medication did not have a significant effect on the changes in vital signs, although trends in NT pro-BNP levels showed higher ascending trends in patients using renin angiotensin system (RAS) inhibitors (median 8.7\%, IQR 3.1-37.2\%) compared with patients who did not use RAS inhibitors (median $0.2 \%$; IQR $-10.5 \%$ to $14.1 \%$; $\mathrm{p}=0.024)$. NT pro-BNP levels also showed higher ascending trends in patients with congestive heart disease (median 23.1\%, IQR 5.2$37.1 \%$ ) compared with patients without (median $1.0 \%$, IQR $-10.2 \%$ to $13.0 \% ; \mathrm{p}=0.006)$. Patients using diuretics also had higher ascending trends in NT pro-BNP levels (median 14.8\%, IQR 1.7-41.2\%) compared with patients without diuretics (median $0.2 \%$, IQR $-11.3 \%$ to $10.9 \%$; $\mathrm{p}=0.004$ ). However, patients using diuretics (median 1.0 L, IQR 0.5-1.0 L) received less fluid resuscitation (median 1.5 L, IQR 1.0-2.1 L; p=0.004). The change in body temperature was not affected by the antipyretic effect of NSAIDs. However, in patients using paracetamol, an ascending trend in body temperature (median $0.8 \%$, IQR $-1.8 \%$ to $1.8 \%$ ) was observed, while patients without paracetamol showed a descending trend in body temperature (median $-1.4 \%$, IQR $-2.8 \%$ to $0.3 \%$; $\mathrm{p}=0.021$ ).

The use of antibiotics prior to ED presentation did not affect the response to treatment of the infection parameters (leucocytes, CRP); neither was the leucocyte

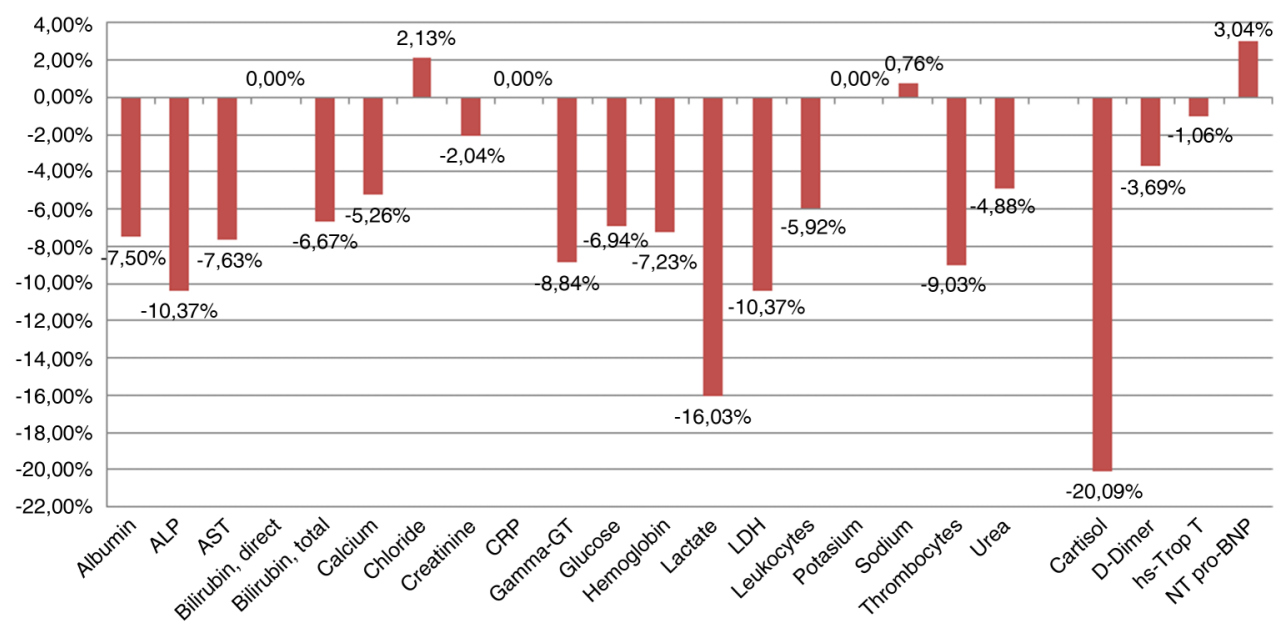

Figure 2 The $\delta$ in biomarker levels between $T_{0}$ and $T_{1} \cdot \gamma$-GT, $\gamma$-glutamyl transferase; ALP, alkaline phosphatase; AST, aspartate transaminase; CRP, $\mathrm{C}$ reactive protein; $\mathrm{CV}$, averaged interassay and intra-assay coefficient of variation during the study inclusion period; Hb, haemoglobin; hs-Trop T, high-sensitivity troponin T; LDH, lactate dehydrogenase; NT pro-BNP, N-terminal prohormone of brain natriuretic peptide. 
response associated with the use of immunosuppressive medication. However, users of immunosuppressive medication (median $3.7 \%$, IQR $-4.8 \%$ to $43.0 \%$ ) showed a tendency of ascending CRP levels (median $-1.4 \%$, IQR $-40.1 \%$ to $-5.8 \%$; $p=0.017$ ). An effect of immunosuppressive or other medication on cortisol levels was not found.

Patients with congestive heart failure showed less decrease in heart rate (median $1.4 \%$, IQR $-3.8 \%$ to $7.8 \%$; vs median $-10.3 \%$, IQR $-16.5 \%$ to $-4.2 \%$; $\mathrm{p}=0.006)$ and had an increasing requirement for supplemental oxygen (median 2.0 L, IQR 2.0-3.0 L; vs median 0.0 L, IQR 0.0-2.0 L; $\mathrm{p}=0.011$ ). We did not find an association between chronic obstructive pulmonary disease (COPD) and the response in oxygen saturation or need for supplemental oxygen. Patients with metastasised tumours tended to have increasing D-dimer levels (median $4.4 \%$, IQR $-1.7 \%$ to $19.4 \%$; vs median $-4.7 \%$, IQR $-16.2 \%$ to $3.0 \%$; $\mathrm{p}=0.023)$.

\section{DISCUSSION}

We performed a pilot study aimed at detecting trends in vital signs and biomarker levels during the early resuscitation of patients with sepsis in the ED. To the best of our knowledge, no other studies have analysed trends in vital signs and routine biomarker levels during resuscitation in the ED. Nowak et $a l^{16}$ recently reported the registration of vital sign data in the first $4 \mathrm{~h}$ in the ED, but these data were neither analysed nor reported.

We found a generally descending trend in most of the vital signs and biomarker levels during the patient's resuscitation in the ED. We specifically noticed descending trends in blood pressure, despite volume therapy. We observed this trend also in other (yet unpublished) studies in our ED. Paradoxically, the patients seem to improve despite this descending trend. This is supported by the relatively low in-hospital and 28-day mortality in our study of $5.1 \%$ and $3.0 \%$, respectively. (Two patients died after $>28$ days in the hospital.) We can only speculate on the mechanism behind this seemingly paradoxical trend in blood pressure. During further analysis, we found that the use of antihypertensive or diuretic medication prior to ED admittance, as well as a history of congestive heart failure or myocardial infarction, did not explain the decrease in blood pressure. While patients with congestive heart failure did show less decrease in heart rate, the use of $\beta$-blockers did not affect the change in heart rate. The use of paracetamol prior to ED presentation led to an increasing trend in body temperature, perhaps while the antipyretic effect of paracetamol has worn off during the patient's stay in the ED. The descending trend in cortisol levels could not be explained by comorbidities or medication use prior to ED presentation; therefore, it is likely that it is partly influenced by its circadian rhythm and partly by the reduction of bodily stress as a response to treatment.
We found only a few ascending trends; we speculate that these ascending trends might be a direct result of the treatment modalities. The only vital sign that showed an ascending trend was the peripheral oxygen saturation, which is most likely caused by supplementation of oxygen, reflected by higher amounts of supplemental oxygen at $T_{1}$. Patients with a history of congestive heart failure showed an increasing oxygen need, while a history of COPD did not explain the additional oxygen requirement. The biomarkers that showed an ascending trend were sodium, chloride and NT pro-BNP. The increase in sodium and chloride levels can easily be explained by the patients receiving intravenous saline solution ( $\mathrm{NaCl} 0.9 \%)$. This might also explain the increase in NT pro-BNP caused by the increased ventricular volume expansion of the heart. On the other hand, there might also be a direct association between NT pro-BNP and the systemic inflammatory response. ${ }^{17}$ Furthermore, we found ascending trends in NT pro-BNP levels in patients using RAS inhibitors or diuretics, although patients using diuretics received less fluid resuscitation, which might suggest that they had earlier volume expansion of the heart.

\section{Limitations}

The main limitation of our pilot study is that it was not designed to detect the cause of the trends: trends might or might not have evolved as a result of the treatment provided. Detected trends could be influenced by several factors such as comorbidity, medication use prior to ED presentation, treatment parameters, dilution effects (by intravenous fluids), variation in laboratory analyses or circadian rhythms. We performed post hoc tests to explore influences of comorbidities and medication use prior to ED presentation in our pilot population, as described above. Dilution might play a role, but we would expect a more even distribution over the different biomarkers when the effects were mainly caused by dilution. Of the measured biomarkers, only cortisol has a well-known circadian rhythm. The variance in laboratory analyses is unlikely to entirely explain the trends, as reflected by the average coefficient of variance during the study's inclusion period shown in table 1 . All factors aforementioned need to be taken into account in further research. Once the clinical value of the trends has been analysed, they can potentially serve as a guide for treatment or to measure disease activity.

\section{Recommendations}

In our pilot study design, we chose an arbitrary interval for the vital sign measurements and repeated blood draw of $3 \mathrm{~h}$. Although trends became apparent during this time frame, the interval might not be the optimal one. We recommend that follow-up studies should determine the optimal interval, with either shorter or longer intervals between repeated measurements. We are currently running a follow-up study in patients with sepsis to detect trends in vital signs measured in 5 min intervals 
during their stay in our ED. In this follow-up study, we explore the course of vital sign changes in more detail. Furthermore, we are in the process of designing a new study, using the results of this pilot study, in which we will continuously record the patient's vital signs beat-to-beat during the first $48 \mathrm{~h}$ in the hospital. The latter study should provide valuable insight into the trends and variability of vital signs in patients with sepsis and potentially provide an early warning of patient deterioration. ${ }^{12}$ Vital signs could also potentially be used to titrate the amount of fluid resuscitation and supplemental oxygen.

The routine biomarkers measured in this pilot study did, in general, only show relatively minor changes during the measurement interval. This makes them less suitable as a response to treatment parameter. The measured study-specific biomarkers, except hs-Trop $\mathrm{T}$, showed larger changes during the measurement interval. We recommend further research to explore their specific responses to treatment. We expect that NT pro-BNP could be a parameter to measure response to fluid resuscitation and might in the future be used to titrate the amount of fluids given. Furthermore, cortisol could be a parameter to measure the body's stress level in response to treatment. The levels of D-dimer could provide information about the status of the coagulation system and disease activity, especially in patients with metastasised tumours.

On the basis of our results, we recommend further exploration of the use of vital signs as a response to treatment parameter in sepsis. They relatively show the largest changes within the measurement interval and are furthermore easily, cheaply and non-invasively measurable.

We expect that trends with a decrease in heart rate, respiratory rate and temperature, as well as an increase in oxygen saturation and blood pressure, could be valued as a positive response to treatment in patients with sepsis, although this pilot study could not (yet) confirm this assumption.

Acknowledgements The authors thank the nurses and physicians in their emergency department for their assistance during the acquisition of the data. They thank the clinical chemists of their central laboratory for providing them with the coefficient of variation values for each biomarker.

Contributors VMQ designed the study, assisted with data acquisition, carried out data analysis and drafted the manuscript. MvM and JCtM participated in the study design, assisted with data interpretation and revised the manuscript. JJML participated in the study design, assisted with data interpretation, revised the manuscript and has given final approval of the version to be published.

Competing interests None declared.
Ethics approval The Institutional Review Board of the University Medical Center Groningen approved the study (METc 2013/297).

Provenance and peer review Not commissioned; externally peer reviewed.

Data sharing statement No additional data are available.

Open Access This is an Open Access article distributed in accordance with the Creative Commons Attribution Non Commercial (CC BY-NC 4.0) license, which permits others to distribute, remix, adapt, build upon this work noncommercially, and license their derivative works on different terms, provided the original work is properly cited and the use is non-commercial. See: http:// creativecommons.org/licenses/by-nc/4.0/

\section{REFERENCES}

1. Yealy DM, Kellum JA, Huang DT, et al., ProCESS Investigators. A randomized trial of protocol-based care for early septic shock. N Engl J Med 2014;370:1683-93.

2. Rivers E, Nguyen B, Havstad S, et al. Early goal-directed therapy in the treatment of severe sepsis and septic shock. $N$ Engl J Med 2001;345:1368-77.

3. Zijlstra J, Monteban W, Meertens J, et al. Septic shock therapy: the recipe or the cook? Crit Care Med 2006;34:2870; author reply 2870-1.

4. Mouncey PR, Osborn TM, Power GS, et al. Trial of early, goal-directed resuscitation for septic shock. N Engl J Med 2015;372:1301-11.

5. Marshall JC, Vincent JL, Guyatt G, et al. Outcome measures for clinical research in sepsis: a report of the 2nd Cambridge Colloquium of the International Sepsis Forum. Crit Care Med 2005;33:1708-16.

6. Dellinger RP, Levy MM, Rhodes A, et al., Surviving Sepsis Campaign Guidelines Committee including The Pediatric Subgroup. Surviving Sepsis Campaign: international guidelines for management of severe sepsis and septic shock. 2012. Intensive Care Med 2013;39:165-228.

7. Samraj RS, Zingarelli B, Wong HR. Role of biomarkers in sepsis care. Shock 2013;40:358-65.

8. Pierrakos C, Vincent JL. Sepsis biomarkers: a review. Crit Care 2010;14:R15.

9. Angus DC, Linde-Zwirble WT, Lidicker J, et al. Epidemiology of severe sepsis in the United States: analysis of incidence, outcome, and associated costs of care. Crit Care Med 2001;29:1303-10.

10. Mason S, Weber EJ, Coster J, et al. Time patients spend in the emergency department: England's 4-hour rule-a case of hitting the target but missing the point? Ann Emerg Med 2012;59:341-9.

11. Geelhoed GC, de Klerk NH. Emergency department overcrowding, mortality and the 4-hour rule in Western Australia. Med J Aust 2012;196:122-6.

12. Buchan CA, Bravi A, Seely AJ. Variability analysis and the diagnosis, management, and treatment of sepsis. Curr Infect Dis Rep 2012;14:512-21.

13. Levy MM, Fink MP, Marshall JC, et al. International Sepsis Definitions Conference. 2001 SCCM/ESICM/ACCP/ATS/SIS International Sepsis Definitions Conference. Intensive Care Med 2003;29:530-8.

14. Dutch Working Party on Antibiotic Policy. SWAB guidelines for antibacterial therapy of adult patients with sepsis. SWAB 2010. www.swab.nl/richtlijnen/sepsis/en (accessed Feb 2016).

15. Cohen J. Statistical power analysis for the behavioral sciences. Academic press, 2013.

16. Nowak RM, Nanayakkara P, DiSomma S, et al. Noninvasive hemodynamic monitoring in emergency patients with suspected heart failure sepsis and stroke: the PREMIUM registry. West $J$ Emerg Med 2014;15:786-94.

17. Rudiger A, Fischler M, Harpes $P$, et al. In critically ill patients, B-type natriuretic peptide (BNP) and N-terminal pro-BNP levels correlate with C-reactive protein values and leukocyte counts. Int J Cardiol 2008;126:28-31. 